\title{
Analyse Spatiale du Potentiel en eau Souterraine dans le Bassin du Zou au Sud du Benin (Afrique de L'ouest)
}

\author{
Francis Enagnon Oussou, M.A. \\ Laboratoire d'Hydrologie Appliquée, Institut National de l'Eau (INE), \\ Université d'Abomey-Calavi, Bénin \\ Joseph Oloukoi, PhD \\ African Regional Institute for Geospatial Information Science and \\ Technology (AFRIGIST), Obafemi Awolowo University Campus, \\ ILE-IFE, Nigeria \\ Nicaise Yalo, Pr. \\ Moussa Boukari, Pr. \\ Laboratoire d'Hydrologie Appliquée, Institut National de l'Eau (INE), \\ Université d'Abomey-Calavi, Bénin
}

Doi:10.19044/esj.2019.v15n27p402 URL:http://dx.doi.org/10.19044/esj.2019.v15n27p402

\section{Résumé}

Dans le socle granito-gneissique du bassin du Zou, l'accès à l'eau potable est principalement assuré par les aquifères de fractures. Dans l'optique de faciliter l'implantation des forages, cette étude d'une part a établi la carte de fractures et d'autre part évalué le potentiel en eau souterraine dans cette partie du Bénin. D'abord les filtres directionnels Sobel sont utilisés pour extraire une représentation du réseau de fractures. De ces données, trois familles de directions majoritaires sont identifiées : N180-N30, N90-N110 et N60-N70. D'autres minoritaires notamment N130-N140 affectent aussi ces formations cristalline et cristallophyllienne. Les longueurs de fractures se concentrent dans un intervalle de 1 à $2 \mathrm{~km}(65 \%)$ et les extrêmes inférieurs à $1 \mathrm{~km}$ et supérieurs à $2 \mathrm{~km}$ donnent respectivement $12,7 \%$ et $22 \%$. Cet ensemble de direction révèle une densité de fractures moyenne de $2,4 \mathrm{~km} / \mathrm{km}^{2}$ avec des zones telles que le Sud de Iroukou de faible valeur $\left(0,8 \mathrm{~km} / \mathrm{km}^{2}\right)$ et Dassa de forte densité $\left(5,2 \pm 0,9 \mathrm{~km} / \mathrm{km}^{2}\right)$. Ces résultats sont utiles à l'implantation car plus de $60 \%$ des forages situés à moins de $200 \mathrm{~m}$ d'une fracture identifiée est positif. De plus, d'autres cartes telles que l'épaisseur d'altération, la lithologie, le drainage, la hauteur de pluies et la pente sont confrontées à la densité de fractures à l'aide de l'analyse multicritère de décision. Selon la contribution de chaque carte à la disponibilité des eaux souterraines, les poids sont 
respectivement : 0,$158 ; 0,074 ; 0,126 ; 0,302 ; 0,065$ et 0,275 . Il ressort de l'analyse spatiale que le bassin est caractérisé par un potentiel très faible $(9,2$ $\%)$ à faible (18\%) vers Djidja, Gangan et Glazoué, modéré $(24,7 \%)$ dans les environs de Zoudji et Yagbo et bon (27,5 \%) à très bon (20,6 \%) vers Savalou. Le potentiel en eau de ces blocs d'aquifères peut varier de façon significative même à l'échelle locale. Malgré les contraintes, ces ressources sont essentielles à l'atteinte de l'Objectif de Développement Durable 6 dans le bassin, bien entendu après une étude approfondie des aquifères afin de garantir l'approvisionnement en eau potable des populations et la pérennité des forages.

Mots-clés: Bassin du Zou, Fractures, Potentiel en eau souterraine, Analyse spatiale, Approvisionnement en eau potable

\title{
Spatial Analysis of Groundwater Potential in Zou Basin (South Benin,West Africa)
}

\author{
Francis Enagnon Oussou, M.A. \\ Laboratoire d'Hydrologie Appliquée, Institut National de l'Eau (INE), \\ Université d'Abomey-Calavi, Bénin \\ Joseph Oloukoi, PhD \\ African Regional Institute for Geospatial Information Science and \\ Technology (AFRIGIST), Obafemi Awolowo University Campus, \\ ILE-IFE, Nigeria \\ Nicaise Yalo, Pr. \\ Moussa Boukari, Pr. \\ Laboratoire d'Hydrologie Appliquée, Institut National de l'Eau (INE), \\ Université d'Abomey-Calavi, Bénin
}

\begin{abstract}
In the granite-gneiss basement of the Zou basin, drinking water access is mainly provided by fracture aquifers. In order to facilitate the borehole drilling, this study, on one hand, established the fracture map and on the other hand evaluated the groundwater potential in this part of Benin. First, Sobel directional filters are used to extract a representation of the fracture network. From these data, three majority directions are identified: N180-N30, N90N110 and N60-N70. Other minority groups, in particular, N130-N140, also affect these crystalline and crystallophyllian formations. The fracture lengths
\end{abstract}


are concentrated in a range of 1 to $2 \mathrm{~km}(65 \%)$ and the extreme values lower than $1 \mathrm{~km}$ and greater than $2 \mathrm{~km}$ are respectively $12.7 \%$ and $22 \%$. This set of direction reveals an average fracture density of $2.4 \mathrm{~km} \cdot \mathrm{km}^{-2}$ with areas such as the South of Iroukou having low values $\left(0.8 \pm 0.9 \mathrm{~km} . \mathrm{km}^{-2}\right)$ and high values in Dassa $\left(5.2 \pm 0.9 \mathrm{~km} . \mathrm{km}^{-2}\right)$. These results are useful for drilling because more than $60 \%$ of boreholes located within $200 \mathrm{~m}$ of an identified fracture is positive. In addition, other maps such as alteration thickness, lithology, drainage, rainfall, and slope are confronted with fracture density using multicriteria decision analysis. Based on the contribution of each map to groundwater availability, the weights are $0.158,0.074,0.126,0.302,0.065$ and 0.275 , respectively. It turns out after spatial analysis that the basin is characterized by a very low $(9.2 \%)$ to low (18\%) potential towards Djidja, Gangan and Glazoué, moderate $(24.7 \%)$ in the Zoudji and Yagbo and good $(27.5 \%)$ to very good $(20.6 \%)$ around Savalou. The water potential of these blocks of aquifers can change significantly even at the local scale. Despite the constraints, these resources are essential to achieving the Sustainable Development Goal 6 in the basin, of course after a thorough study of the aquifers in order to guarantee drinking water supply of the populations and the durability of the boreholes.

Keywords: Zou basin, Fractures, Groundwater potential, Spatial analysis, Drinking water supply

\section{Introduction}

L'eau souterraine est une ressource naturelle vitale pour un approvisionnement en eau potable sûr et économique en milieu urbain et rural (Magesh et al., 2012). En effet au Bénin et particulièrement dans le bassin du Zou, le respect de l'article 6 de la Loi $\mathrm{n}^{\circ} 2010-44$ portant gestion de l'eau est assuré grâce à l'exploitation des réservoirs souterrains à l'exception des zones hydrogéologiques difficiles (Boukari, 1998). Selon les conclusions du document de Vision Eau pour le Bénin à l'horizon 2025, moins de $18 \%$ des ressources en eaux souterraines seront sollicité pour couvrir la demande en eau des populations dans les différentes branches d'activités (Vision Eau Bénin 2025, 1998). La satisfaction de cette demande estimée à $927000 \mathrm{~m}^{3}$ d'eau par jour en milieu urbain et rural pour l'approvisionnement en eau potable à l'horizon 2025 exige forcement une analyse succincte du potentiel en eau souterraine. Aussi, il s'agit de travailler à réduire considérablement le taux de forages négatifs. En vue d'atteindre cette ambition, l'étude de la disponibilité de la ressource s'avère importante (Sreedhar et al., 2009 ; Abdalla, 2012).

À l'instar de plusieurs travaux tels que Kanohin et al. (2012); Magesh et al. (2012) ; Ndatuwong et Yadav (2014) ; Oikonomidis et al. (2015), l'étude $\mathrm{du}$ potentiel en eau souterraine tient compte des facteurs géologiques, 
hydrologiques et climatiques. Les aquifères particulièrement intéressants pour l'approvisionnement en eau dans ces zones se situent dans les couches altérées et fissurées/fracturées des roches cristallines ou cristallophylliennes (Castany, 1982 ; Banton et Bangoy, 1997). Il fut reconnu depuis des années que même dans les régions géologiquement bien connues, les images satellitaires apportent des données nouvelles, non seulement en faisant apparaitre de grandes unités lithologiques, mais aussi en montrant des failles et des fractures qui ne sont pas connues (Chorowicz, 1984). La télédétection apparaît comme un outil puissant de prospection pouvant contribuer de façon significative aux projets géologiques et au développement soutenable car beaucoup d'informations identifiées par les satellites se sont indirectement révélées très utiles (Colloque de Géologie Africaine, 2006 ; Meijerink, 2007). Les travaux de Becker (2006) ont montré que plusieurs capteurs tels que ETM+, RADARSAT-1, ASTER, GRACE et autres rapportent des données potentiellement utilisables pour l'investigation des eaux souterraines.

Par ailleurs, des écrits élucident l'état des lieux des ressources en eau au centre Bénin en particulier dans le bassin du Zou (Boukari, 1998 ; Vissin, 1998 ; Boko, 2004 ; Awoyé, 2007 ; Bossa, 2007 ; Kodja et al., 2012 ; Sintondji et al., 2013 ; etc.). Cependant une analyse de la disponibilité des eaux souterraines actualise l'état des lieux et met l'accent sur l'aptitude hydrogéologique du bassin. Dans le cadre de l'analyse du potentiel en eau souterraine, le nombre de facteurs utilisés, les seuils considérés pour la reclassification et la pondération ne sont pas standards. Une adaptation est généralement faite en considérant les conditions climatique et géologique du milieu étudié. Ainsi, cette étude utilise la densité de linéament, l'épaisseur d'altération, la lithologie, la pluviométrie, le drainage et la topographie pour évaluer la répartition spatiale de la disponibilité en eau souterraine du bassin du Zou.

\section{Materiel et methodes \\ Milieu d'étude}

Le bassin du Zou est situé entre les latitudes $7^{\circ}$ et $8^{\circ} 33^{\prime}$ Nord et les longitudes $1^{\circ} 35^{\prime}$ et $2^{\circ} 30^{\prime}$ Est. D'une superficie d'environ $8491 \mathrm{~km}^{2}$, il déborde légèrement dans le territoire togolais $(2,24 \%)$ au Centre-Ouest du Bénin (Figure 1). Il couvre entièrement et en partie quatre communes (Bantè, Glazoué, Savalou, Dassa) des Collines et six communes du Zou (Djidja, ZaKpota, Bohicon, Covè, Zagnanado, Ouinhi). 


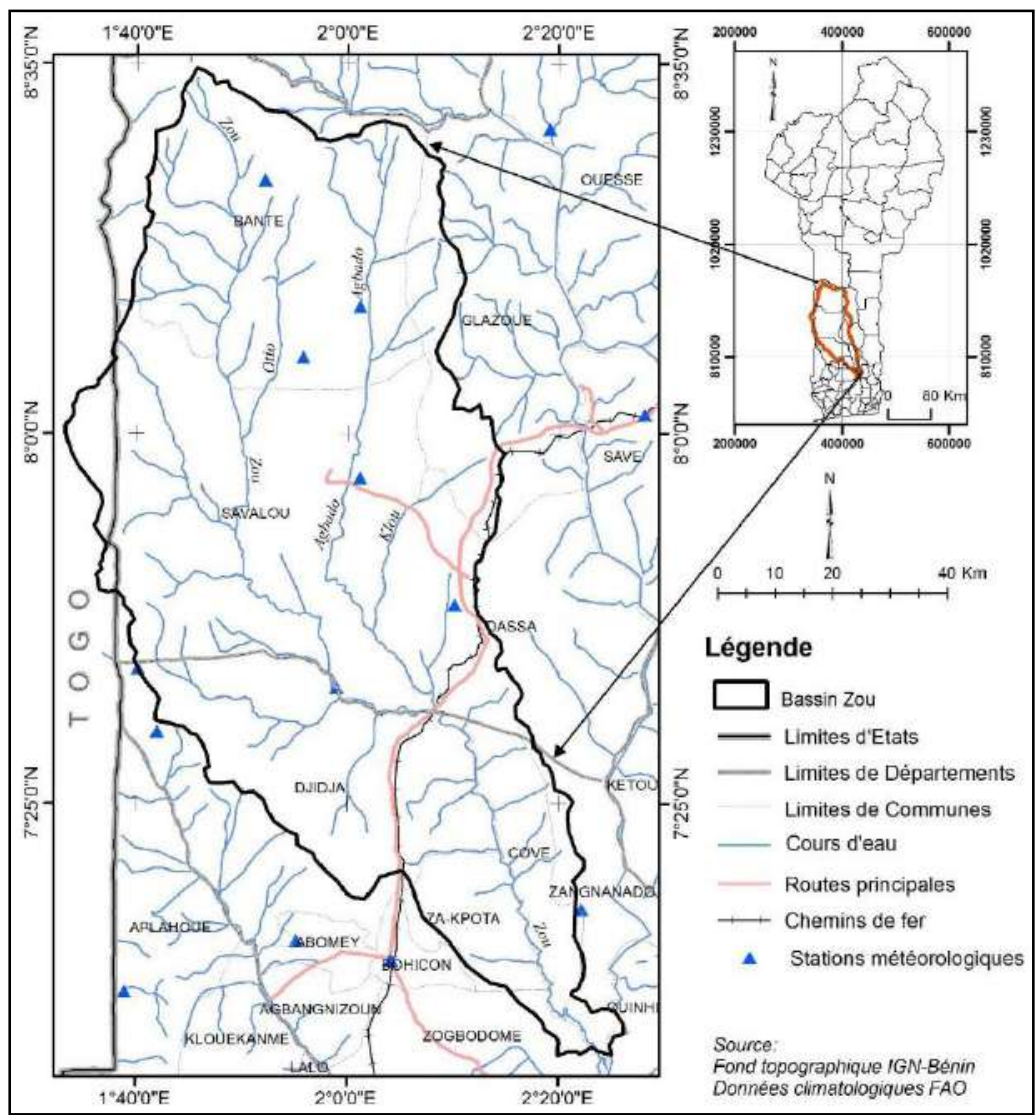

Figure 1. Situation géographique du bassin versant du Zou.

Le climat dans cette zone du centre Bénin est intermédiaire entre le climat sub-équatorial de la côte et le climat soudano sahélien du Nord Bénin (Houssou, 1998). Il constitue pour l'essentiel une zone où s'estompent les influences de la mousson du sud-ouest et de l'alizé continental appelé harmattan du nord-est. La moyenne de la pluviométrie annuelle sur 30 ans avoisine $1139 \mathrm{~mm}$ (Figure 2). Le mois de juillet est le plus arrosé $(172 \mathrm{~mm}$ environ) tandis que la hauteur de pluies peut descendre jusqu'à $18 \mathrm{~mm}$ en décembre. Le maximum de température est enregistré en février avec une moyenne de $29,8{ }^{\circ} \mathrm{C}$ et le minimum en août $\left(25^{\circ} \mathrm{C}\right.$ en moyenne). Le capteur OLI-TIRS à bord du Landsat 8 a mesuré le 28 décembre 2014 une température moyenne de $24{ }^{\circ} \mathrm{C}$ pour un écart type de $1,3{ }^{\circ} \mathrm{C}$. 


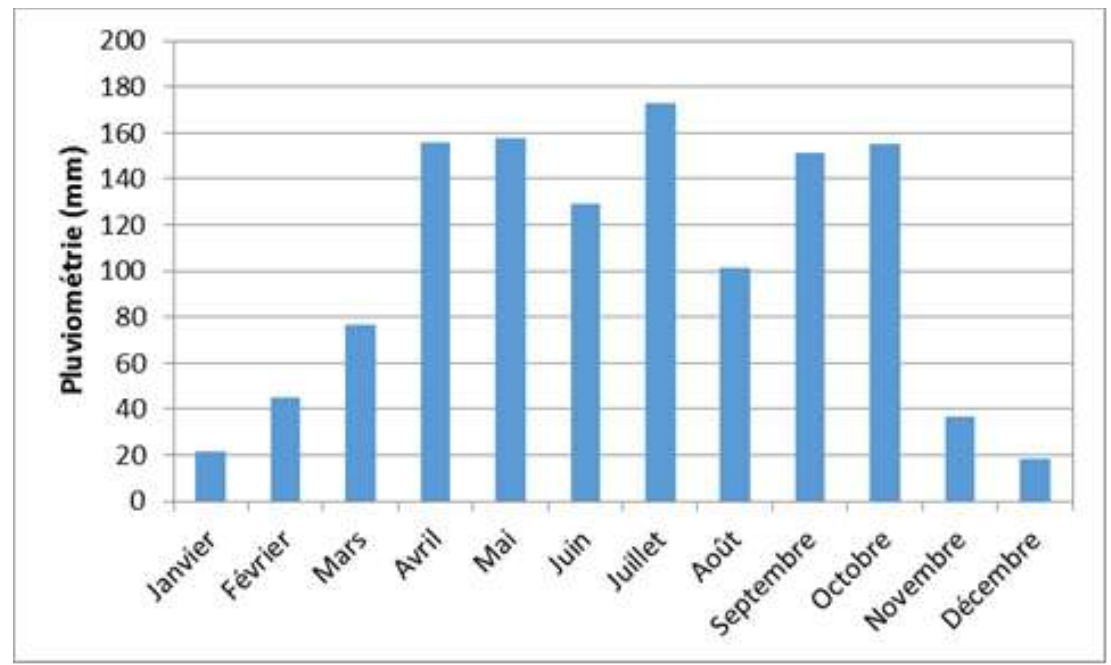

Figure 2. Diagramme pluviométrique (Station de Bohicon de 1985 à 2015)

Alidou et al., (1975), Boukari (1982) et autres ont montré la complexité géologique de cette zone du Bénin, tant à l'échelle locale que régionale. Il s'agit en effet d'ensembles lithologiques et structuraux ayant subi plusieurs phases de déformation, de métamorphisme et de magmatisme (Figure 3). Le bassin versant du Zou s'étend sur une surface modelée par des roches très anciennes datant du précambrien (le socle granito-gneissique). Sur ce modelé de socle d'altitude moyenne variant entre 29 et $284 \mathrm{~m}$, s'individualise une série d'affleurement rocheux (inselbergs) ou chaînes de collines, isolées et dénudées : c'est surtout le cas des collines de Savalou et de Dassa (Okioh, 1972). 


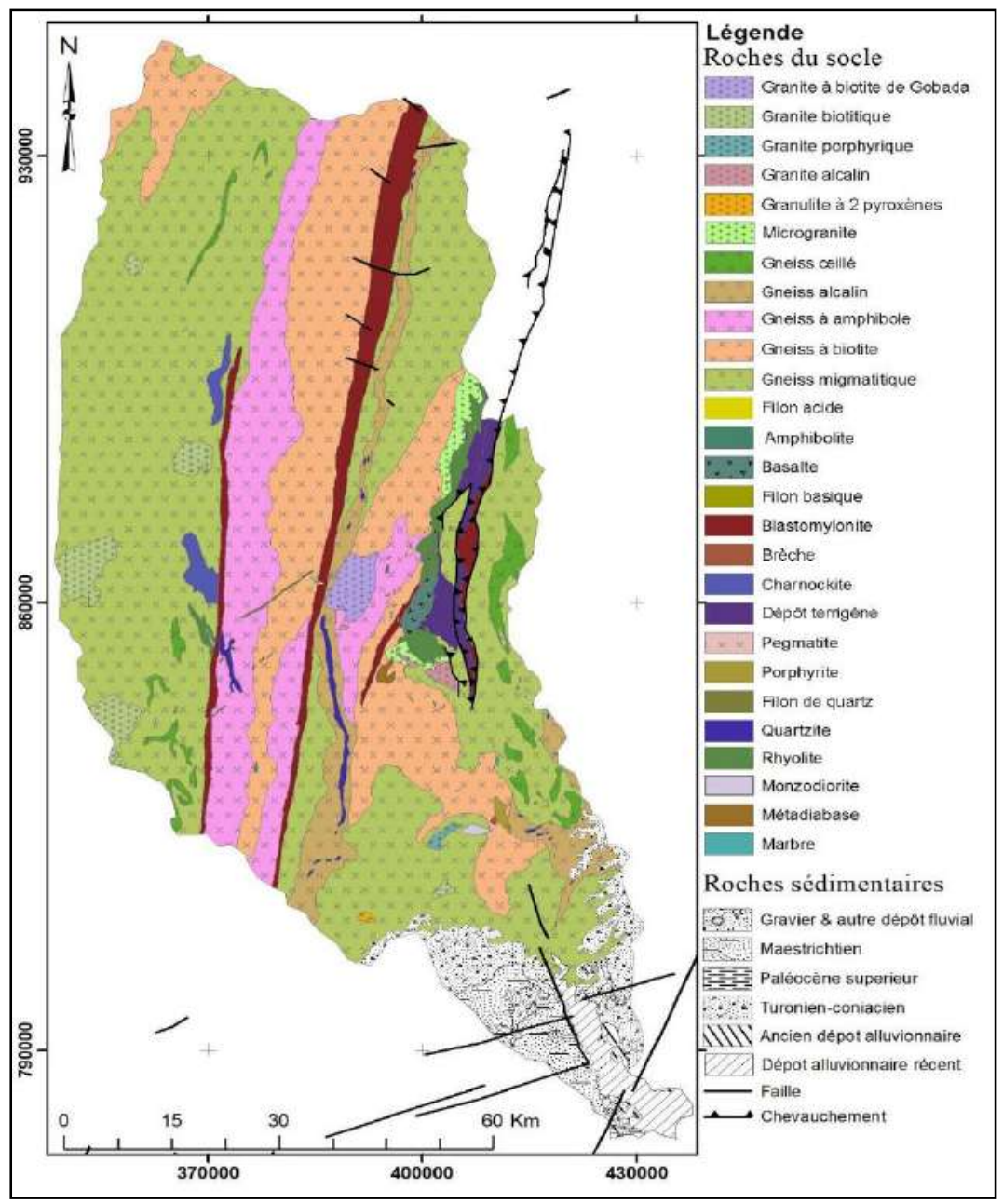

Figure 3. Carte géologique du bassin du Zou-

Source : carte géologique du Bénin au 1/200 000 (OBEMINES). Projection UTM 31. Ellipsoïde CLARKE 1880

\section{Données}

Deux scènes de path 192 et row 54-55, de résolution $30 \mathrm{~m}$ en mode multi-spectral et $15 \mathrm{~m}$ en mode panchromatique, ont été téléchargées sur le site américain http://glcf.umd.edu/data/. Ces données sont acquises le 28 décembre 2014 par le capteur OLI-TIRS à bord du Landsat 8. Les images ont permis d'identifier les linéaments de la zone d'étude. L'image Shuttle Radar Topography Mission (SRTM) de résolution $30 \mathrm{~m}$ a été téléchargée sur le site américain http://www.earthdata.nasa.gov. Le Modèle Numérique de Terrain fait référence à des fichiers matriciels fournis par deux agences américaines que sont la NASA et la NGA (ex-NIMA). La mission SRTM (Shuttle Radar Topography Mission), lancée en 2000 a recueilli des données altimétriques sur une grande partie de la Terre par interférométrie d'images RSO (Rabus et al., 
2003; Jarvis et al., 2004). L'image a été utilisée pour calculer la pente afin de restituer la topographie de la zone d'étude et analyser son influence sur la disponibilité de l'eau souterraine. Quatre feuilles topographiques au 1/200000 (NB31-XX, NB31-XXI, NC31-II et NC31-III) couvrent entièrement le bassin et ont servi à l'extraction des informations du fond de carte pour la production des cartes thématiques. Les cours d'eau extraits ont été utilisés pour calculer le drainage. La carte géologique au 1/200000 de la zone a été géoréférencée et digitalisée pour extraire les limites des roches (lithologies) et les failles. Les données de pluie des stations (Bantè, Tchetti, Savalou, Savè, Bohicon, Pobè, Abomey, Agouna, Dassa, Zagnanado, Gouka, Pira et Aklankpa) de la zone d'étude ont été utilisées pour réaliser la carte des isohyètes. Enfin les données d'épaisseur d'altération mesurées sur plus de 500 forages obtenus à partir de la Base de Donnée Intégrée (BDI) de la Direction Générale de l'Eau (DG-Eau) ont contribué à une estimation de la distribution spatiale à l'échelle du bassin et la validation de la carte du potentiel en eau.

\section{Méthodes}

Il est considéré comme linéament tout alignement récupérable sur les photographies aériennes ou sur les images satellitaires. Les traces considérées sont assimilables soit à des limites géologiques, ruptures de pente, modifications dans le tracé d'un cours d'eau ou d'une structure quelconque, variations de faciès et les zones fracturées (Biémi, 1992 ; Meijerink, 2007 ; Bruning et al., 2009). Dans les projets de prospection d'eau souterraine en région de socle, la recherche et l'analyse des linéaments structuraux représentent une option principale. Plusieurs études (Boussari, 1975 ; Boukari, 1982 ; Jourda, 1987 et Kouassi et al., 2013) effectuées dans les socles cristallin et cristallophyllien de l'Afrique de l'Ouest ont prouvé que les zones productives se localisent généralement aux environs de ces accidents géologiques. Les images de Télédétection fournissent une vue synoptique de grande zone et offre d'importantes possibilités d'analyse. Ainsi, les images Landsat OLI-TIRS obtenues ont subi les opérations telles que l'amélioration $\mathrm{du}$ contraste, le pan-sharpening et l'analyse en composante principale. L'opération de Pan-charpenning signifie l'utilisation d'une image panchromatique (bande unique) pour améliorer une image multispectrale. Cette opération a permis d'accéder à un niveau de détail meilleur du point de vue spatial et radiométrique. L'analyse en composante principale est une transformation orthogonale d'image. L'information contenue dans les 10 bandes du Landsat/OLI-TIRS est regroupée dans un nombre restreint de néobandes (composante principale 1).

Le filtrage d'image est une modification des fréquences spatiales (contrastes locales) où l'on transforme la valeur radiométrique d'un pixel considéré par rapport à ses voisins. C'est une opération qui utilise la matrice 
de convolution pour rehaussement certains détails spécifiques d'une image. Le concept de convolution indique une application qui exprime une combinaison des tons de gris d'un pixel en rapport avec ceux de ces voisins. Le but du filtrage d'image est essentiellement de supprimer les bruits, atténuer ou accentuer les fréquences spatiales en occurrence les hautes fréquences telles que les linéaments en vue de rendre plus nets les thèmes ou les composantes de deux ou plusieurs thèmes contigus pour une bonne interprétation visuelle de l'image. Les segments sont assimilables soit à des limites géologiques, ruptures de pente, modifications dans le tracé d'un cours d'eau ou d'une structure quelconque, variations de faciès et quelquefois aussi les zones fracturées (Meijerink, 2007 ; Bruning et al., 2009). Le filtre de SOBEL met en relief les fortes transitions de réflectance et les hautes fréquences spatiales généralement associées aux fractures (Biémi, 1992, Alhassane et al., 2018; Assoma et al., 2018 et Kouassi et al., 2019). Des études ayant des objectifs similaires (Youan Ta et al. 2008 ; Kouame, 2009 et Kouassi, et al., 2018) ont utilisé le filtre SOBEL soit pour l'analyse structurale soit pour la prospection des eaux souterraines. Dans le cadre de nos recherches, les fractures sont extraites en appliquant le filtre proposé par Kamagaté et al. (2011) à la composante principale 1 (Tableau 1).

Tableau 1. Matrices de convolution 7 X 7 (Kamagaté et al., 2011).

\begin{tabular}{|c|c|c|c|c|c|c|}
\hline \multicolumn{7}{|c|}{ Sobel N-S } \\
\hline 0 & 1 & 1 & 1 & 1 & 1 & 2 \\
\hline-1 & 0 & 2 & 2 & 2 & 3 & 1 \\
\hline-1 & -2 & 0 & 3 & 4 & 2 & 1 \\
\hline-1 & -2 & -3 & 0 & 3 & 2 & 1 \\
\hline-1 & -2 & -4 & -3 & 0 & 2 & 1 \\
\hline-1 & -3 & -2 & -2 & -2 & 0 & 1 \\
\hline-2 & -1 & -1 & -1 & -1 & -1 & 1 \\
\hline
\end{tabular}

Sobel E-O

\begin{tabular}{|c|c|c|c|c|c|c|}
\hline \multicolumn{7}{|c|}{ Sobel NO-SE } \\
\hline-1 & -1 & -1 & 0 & 1 & 1 & 1 \\
\hline-1 & -1 & -2 & 0 & 2 & 1 & 1 \\
\hline-1 & -2 & -3 & 0 & 3 & 2 & 1 \\
\hline-2 & -3 & -4 & 0 & 4 & 3 & 2 \\
\hline-1 & -2 & -3 & 0 & 3 & 2 & 1 \\
\hline-1 & -1 & -2 & 0 & 2 & 1 & 1 \\
\hline-1 & -1 & -1 & 0 & 1 & 1 & 1 \\
\hline
\end{tabular}

\begin{tabular}{|c|c|c|c|c|c|c|}
\hline \multicolumn{7}{|c|}{ Sobel E-O } \\
\hline 1 & 2 & 1 & 1 & 1 & 1 & 0 \\
\hline 1 & 3 & 2 & 2 & 2 & 0 & 1 \\
\hline 1 & 2 & 4 & 3 & 0 & -2 & -1 \\
\hline 1 & 2 & 3 & 0 & -3 & -2 & -1 \\
\hline 1 & 2 & 0 & -3 & -4 & -2 & -1 \\
\hline 1 & 0 & -2 & -2 & -2 & -3 & -1 \\
\hline 1 & -1 & -1 & -1 & -1 & -1 & -2 \\
\hline
\end{tabular}

\begin{tabular}{|c|c|c|c|c|c|c|}
\hline \multicolumn{7}{|c|}{ Sobel NE-SO } \\
\hline 1 & 1 & 1 & 2 & 1 & 1 & 1 \\
\hline 1 & 1 & 2 & 3 & 2 & 1 & 1 \\
\hline 1 & 2 & 3 & 4 & 3 & 2 & 1 \\
\hline 0 & 0 & 0 & 0 & 0 & 0 & 0 \\
\hline-1 & -2 & -3 & -4 & -3 & -2 & -1 \\
\hline-1 & -1 & -2 & -3 & -2 & -1 & -1 \\
\hline-1 & -1 & -1 & -2 & -1 & -1 & -1 \\
\hline
\end{tabular}

Les 4 matrices 7 x 7 sont choisies selon que leur efficacité a été prouvée par Kamagate et al. (2011). Les zones de faiblesse contenues dans le bassin sont ainsi récupérées sous forme de linéaments utile à l'implantation des forages.

L'extraction des linéaments à partir des images filtrées est possible manuellement, mais elle reste subjective et sujette à des erreurs de numérisation. La méthode automatique rend l'opération plus facile, même si 
elle peut être parfois affectée par des biais liés aux techniques précédemment utilisées. Pour l'extraction, nous avons utilisé le module LINE du logiciel PCI Geomatics, en appliquant les valeurs par défaut des paramètres du module d'extraction. Les documents obtenus sont ensuite affichés sur des images à haute résolution (par exemple les images Google Earth) pour vérification et nettoyage.

Le fonctionnement des aquifères de socle conjugue deux fonctions, que sont souvent le rôle de réservoir assuré principalement par les produits de l'altération de la roche en place, développée depuis le niveau du sol jusqu'à parfois plusieurs dizaines de mètres de profondeur. Les épaisseurs d'altération mesurées après réalisation des forages sont interpolées par la méthode de krigeage. Notons que l'objectif visé dans cette spatialisation est d'avoir une idée sur la zonation ou la tendance généralisée des épaisseurs rencontrées dans le bassin. Une reclassification de la couche raster issue de l'interpolation est effectuée et ensuite pondérée (Tableau 2 et 3 ).

La nature lithologique des formations géologiques conditionne très fortement la disponibilité en eau. Le bassin du Zou est partagé entre le Bassin Sédimentaire Côtier (BSC) du Bénin et le socle. Le sédimentaire forme quatre grands aquifères continus que sont le Turonien-Coniacien, le Paléocène, le Continental Terminal et le Quaternaire (Tableau 2). L'état hydrodynamique représenté par la transmissivité $\left(\mathrm{L}^{2} / \mathrm{T}\right)$ de trois aquifères est consigné dans le tableau ci-après :

Tableau 2. Valeur moyenne de transmissivité (Le Barbe et al., 1993 et BURGEAP-BRGM, 1994)

\begin{tabular}{|c|c|}
\hline Formation aquifère & Transmissivité moyenne \\
\hline Turonien-Coniacien & $4.10^{-2}$ à $10^{-2} \mathrm{~m}^{2} / \mathrm{s}$ \\
\hline Paléocène & $10^{-3}$ à $10^{-4} \mathrm{~m}^{2} / \mathrm{s}$ \\
\hline Quaternaire & $10^{-3}$ et $3.10^{-2} \mathrm{~m}^{2} / \mathrm{s}$ \\
\hline
\end{tabular}

Un aquifère du socle est par contre discontinu, c'est-à-dire lié à la fracture qui elle-même est continue de façon locale. L'aquifère qui peut être captée se trouve dans la partie fracturée de la roche saine et/ou dans la couche d'altération. L'état hydrodynamique de chaque formation est pondéré en vue de les ranger dans un ordre de potentialité en eau. La pondération proposée par Magesh et al. (2012) est modifiée et adaptée aux réalités du secteur d'étude (Tableau 2 et 3 ).

La pente est calculée dans l'environnement QGIS en pourcentage. Le résultat obtenu subit une reclassification en tenant comptes des intervalles fixés dans le tableau 3. Le drainage de surface est la suppression ordonnée des excès d'eau à la surface du sol grâce au réseau hydrographique. Le drainage d'un bassin met en relief le mètre linéaire du cours d'eau par unité de surface. Après calcul de la densité, la reclassification et la pondération sont effectuées afin de cibler les aires favorables à l'accumulation des eaux de surface. Les 
intervalles proposés varient d'une étude à une autre et sont adaptés selon les conditions climatiques et géologiques spécifiques du milieu d'étude (Sreedhar et al., 2009 ; Abdalla, 2012 ; Kanohin et al., 2012 ; Magesh et al., 2012 ; Ndatuwong et Yadav, 2014 ; Oikonomidis et al., 2015) (Tableau 3). La carte d'égale valeur de pluie (isohyète) est obtenue à partir des hauteurs de pluies enregistrées au niveau des stations (Bantè, Tchetti, Savalou, Savè, Bohicon, Pobe, Abomey, Agouna, Dassa, Zagnanado, Gouka, Pira et Aklankpa).

Tableau 3. Classification et rang des facteurs.

\begin{tabular}{|c|c|c|}
\hline Facteur & Reclassification & Rang \\
\hline \multirow{5}{*}{$\begin{array}{l}\text { Densité de } \\
\text { fractures } \\
\left(\mathrm{km} / \mathrm{km}^{2}\right)\end{array}$} & $0-0,6$ & 1 \\
\hline & $0,6-1,2$ & 3 \\
\hline & $1,2-1,8$ & 5 \\
\hline & $1,8-2,4$ & 9 \\
\hline & $>2,4$ & 13 \\
\hline \multirow{5}{*}{$\begin{array}{l}\text { Épaisseur du } \\
\text { recouvrement } \\
\text { (m) }\end{array}$} & $0-10$ & 1 \\
\hline & $10-20$ & 3 \\
\hline & $20-30$ & 5 \\
\hline & $30-40$ & 9 \\
\hline & $>40$ & 13 \\
\hline \multirow{5}{*}{ Lithologie } & $\begin{array}{c}\text { Granite, gneiss, migmatite, } \\
\text { basalte, quartz, amphibolite, } \\
\text { pegmatite, porphyroïde et } \\
\text { monzodiorite }\end{array}$ & 1 \\
\hline & $\begin{array}{c}\text { Argiles, marnes, sables et } \\
\text { calcaire du Maestrichtien ; } \\
\text { marbre }\end{array}$ & 3 \\
\hline & $\begin{array}{c}\text { Faciès argilo-marneux et } \\
\text { calcaire } \\
\text { de l'Éo-Paléocène }\end{array}$ & 9 \\
\hline & Alluvions (sables et graviers) & 13 \\
\hline & $\begin{array}{c}\text { Faciès sableux } \\
\text { du Turonien-Coniacien }\end{array}$ & 25 \\
\hline \multirow{5}{*}{$\begin{array}{l}\text { Densité de } \\
\text { drainage } \\
\left(\mathrm{km} / \mathrm{km}^{2}\right)\end{array}$} & $0,00-0,05$ & 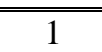 \\
\hline & $0,05-0,10$ & 3 \\
\hline & $0,10-0,15$ & 5 \\
\hline & $0,15-0,20$ & 9 \\
\hline & $>0,20$ & 13 \\
\hline \multirow{5}{*}{$\begin{array}{l}\text { Précipitations } \\
\text { annuelles } \\
\text { moyennes }(\mathrm{mm})\end{array}$} & $<<1080$ & 3 \\
\hline & $1080-1100$ & 5 \\
\hline & $1100-1120$ & 7 \\
\hline & $1120-1140$ & 9 \\
\hline & $>1140$ & 13 \\
\hline \multirow{3}{*}{$\begin{array}{l}\text { Pente (en } \\
\text { pourcentage) }\end{array}$} & $<<2$ & 13 \\
\hline & $2-6$ & 9 \\
\hline & $>6$ & 3 \\
\hline
\end{tabular}


A l'aide de l'Analyse Multicritère Critère (AMC) de décision (Saaty, 1971 ; Chevarie et Periche, 2001), les poids sont calculés en faisant une comparaison deux à deux des paramètres (Tableau 4). La matrice issue de l'analyse permet de calculer les vecteurs propres et valeurs propres. Les vecteurs propres sont les poids appliqués à chaque paramètre pour avoir la carte finale.

Tableau 4. Calcul du poids affecté à chaque paramètre.

\begin{tabular}{|c|c|c|c|c|c|c|c|}
\hline $\begin{array}{c}\text { Disponibilité en } \\
\text { eau souterraine }\end{array}$ & Lin & Rec & Lit & Dr & P & S & Poids \\
\hline Lin & 1 & 3 & 5 & 3 & $1 / 2$ & 3 & $\mathbf{0 , 2 7 5}$ \\
\hline Rec & $1 / 3$ & 1 & 3 & 2 & $1 / 3$ & 3 & $\mathbf{0 , 1 5 8}$ \\
\hline Lit & $1 / 5$ & $1 / 3$ & 1 & $1 / 3$ & $1 / 3$ & 2 & $\mathbf{0 , 0 7 4}$ \\
\hline $\mathrm{Dr}$ & $1 / 3$ & $1 / 2$ & 3 & 1 & $1 / 2$ & 2 & $\mathbf{0 , 1 2 6}$ \\
\hline $\mathrm{P}$ & 2 & 3 & 3 & 2 & 1 & 3 & $\mathbf{0 , 3 0 2}$ \\
\hline $\mathrm{S}$ & $1 / 3$ & $1 / 3$ & $1 / 2$ & $1 / 2$ & $1 / 3$ & 1 & $\mathbf{0 , 0 6 5}$ \\
\hline Somme & 4,20 & 8,17 & 15,50 & 8,83 & 3,00 & 14,00 & \\
\hline
\end{tabular}

Lin : linéament. Rec : recouvrement ou épaisseur d'altération. Lit : lithologie.

Dr: drainage. $P$ : pluviométrie. $S$ : pente.

Le ratio de cohérence obtenu est $0,08<10 \%$. La valeur étant inférieure au seuil fixé (10\%), la matrice de départ est validée.

La somme des produits des six cartes reclassifiées $(\mathrm{Ci})$ par les poids respectifs $(\mathrm{Pi})$ donne une carte synthétique qui retrace le potentiel en eau souterraine du bassin :

$$
P E S=\sum P i \times C i
$$

où $P E S$ est le Potentiel en Eau Souterraine, $P i$ le Poids affecté à une carte $C i$ et $C i$ les cartes représentant les paramètres utilisés.

$$
P E S=\sum(\text { Lin }, \text { Rec }, \text { Lit }, \text { Dr }, P, S)
$$

Lin : Linéament, Rec : Recouvrement ou épaisseur d'altération, Lit : Lithologie, $D r$ : Drainage, $P$ : Pluviométrie, $S$ : Pente

\section{Resultats}

La figure 4 présente la carte de fractures obtenue après les divers rehaussements et l'application des filtres Sobel. Le nombre de segment identifiés avoisine 5136 pour une longueur moyenne de $1,6 \mathrm{~km}$ et un écart type de $0,9 \mathrm{~km}$. En outre $12,7 \%$ des longueurs de fractures est inférieur à $1 \mathrm{~km}, 65$ $\%$ compris entre 1 et $2 \mathrm{~km}$ et $22 \%$ supérieur à $2 \mathrm{~km}$. L'indice du plus proche voisin est 0,67 signifiant que les fractures identifiées sont agrégées. Le score $\mathrm{z}(-110)$ et la valeur $\mathrm{p}$ (inférieure à 0,01) montrent que dans le bassin l'agrégation est statistiquement significative jusqu'à un niveau de confiance de $99 \%$. C'est la preuve que la distribution des fractures n'est pas aléatoire. Le réseau de fracture extrait est ainsi le résultat des actions géologique (accident tectonique) et autres exogènes. Les principales familles de fractures identifiées 
sont N180-N30 (25,8 \%), N90-N110 (17,3\%) et N60-N70 (11,1\%). Ces directions majoritaires donnent lieu aux plus importants réseaux d'écoulement souterrain dans le bassin. D'autres directions minoritaires non négligeables notamment N130-N140 (9,6\%) entretiennent également les débits des forages dans le bassin.

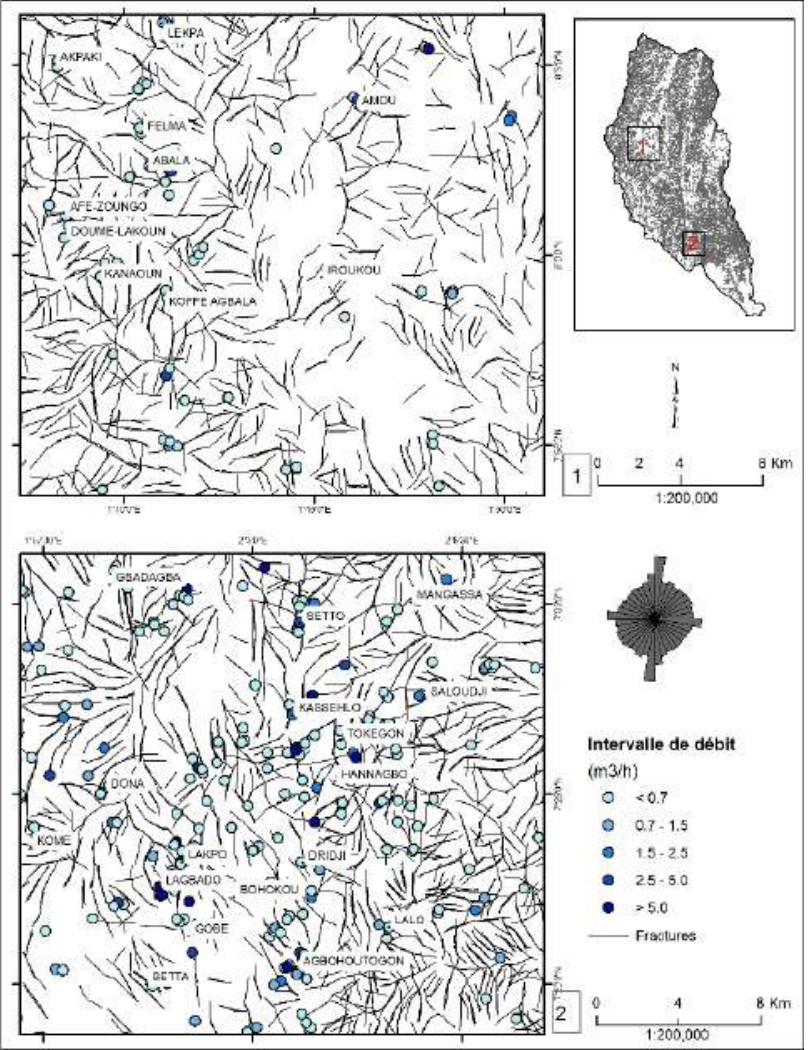

Figure 4. Carte des fractures identifiées versus débit des forages.

La densité de linéament dans le bassin varie entre 0,8 et $5,2 \mathrm{~km} / \mathrm{km}^{2}$ avec une moyenne de $2,4 \mathrm{~km} / \mathrm{km}^{2}$ et un écart type de $0,9 \mathrm{~km} / \mathrm{km}^{2}$ (Figure 5). La zone de chevauchement, l'emprise des cours d'eau ainsi que certaines localités des communes de Savalou, Dassa, Bante et Glazoué affichent de forte densité. Les zones de Lalo, Sowe et environs, au sud de Gbadagba, Gocha, Bantè et au Sud de Djagbalo présentent de faible densité de fractures. La partie $\mathrm{du}$ bassin correspondant au BSC affiche également une densité bien évidemment moindre puisqu'il s'agit des roches sédimentaires. 


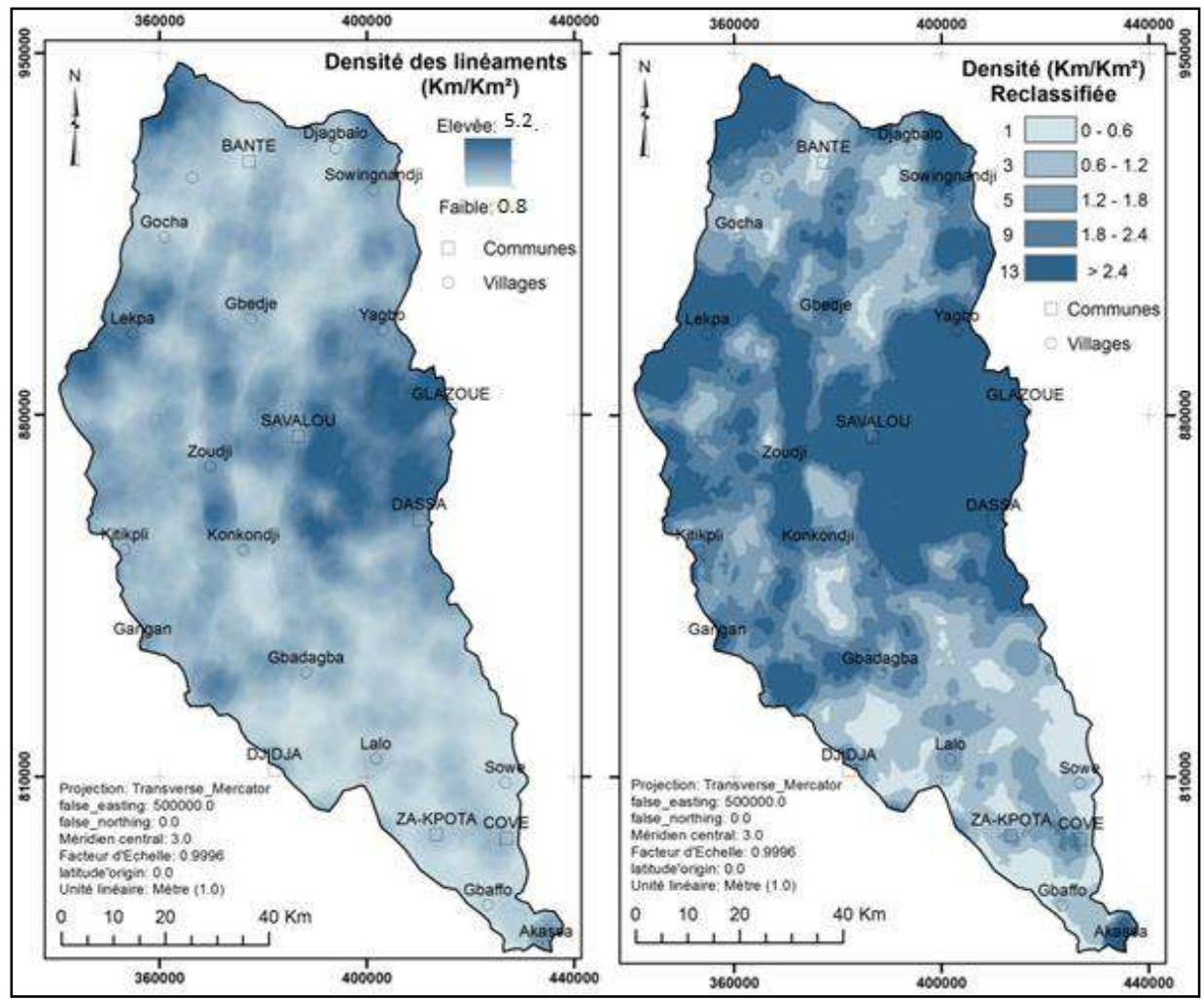

Figure 5. Carte de la densité des linéaments (à gauche) et carte reclassifiée (à droite).

Les valeurs oscillent entre $0 \mathrm{~m}$, au niveau des affleurements, et $65 \mathrm{~m}$, au nord du plateau d'Abomey, dans la partie socle du bassin. L'épaisseur moyenne est de 20,5 m ( $\pm 8,3 \mathrm{~m})$. Dans le bassin, l'aquifère du TuronienConiacien prend le poids le plus élevé (25), ensuite viennent les alluvions du Quaternaire (13), les faciès argilo-marneux de l'Éo-Paléocène (9) et les argiles, marnes, sables et calcaire du Maestrichtien (3).

Plus de $60 \%$ du secteur d'étude a une pente comprise entre $2 \%$ et $6 \%$. Les valeurs les plus élevées (supérieure à 6\%) correspondent aux zones géomorphologiques accidentées comme Savalou, Dassa, Gbadagba et leurs environs. Le drainage estimé après calcul affiche un minimum de $0,1 \mathrm{~km} / \mathrm{km}^{2}$ vers l'ouest (Dassa, Savalou et au nord-ouest de Glazoué), vers l'est (de Lekpa à Kitikpli) et sur le plateau d'Abomey. Le maximum atteint $0,3 \mathrm{~km} / \mathrm{km}^{2}$ vers Gocha, Lougba-Kotakpa et Djagbalo au nord et à l'est de Lalo situés dans l'emprise des cours d'eau. La moyenne spatiale est estimée à $0,13 \mathrm{~km} / \mathrm{km}^{2}$ pour un écart type de $0,048 \mathrm{~km} / \mathrm{km}^{2}$ (Figure 6). La moyenne spatiale de la pluviométrie est $1116 \mathrm{~mm}$ avec un écart type de $23 \mathrm{~mm}$. Le minimum 1028 mm est enregistré à l'ouest, aux environs de Kitikpli et Gangan, et au nord-est, vers Yagbo et Sowingnandji. Le maximum $1179 \mathrm{~mm}$ se localise vers Savalou et Dassa, à l'est, et vers Bantè, au nord. 

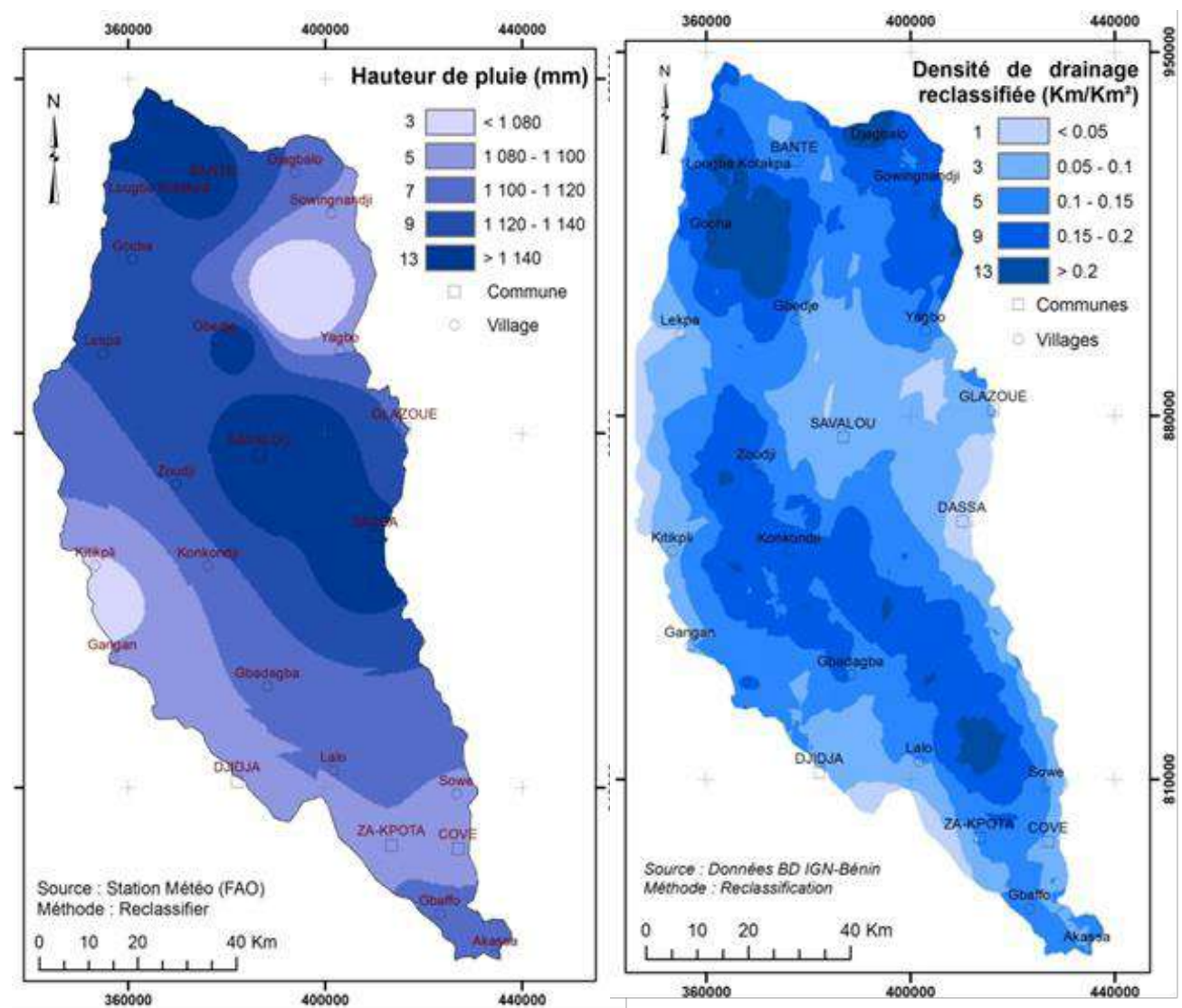

Figure 6. Cartes : pluviosité annuelle moyenne (à gauche) et densité de drainage (à droite)

La validation de la matrice à l'aide du ratio de cohérence a permis de retenir les poids ci-après : 30,2\% (hauteur de pluies), $27,5 \%$ (densité de fractures), 15,8\% (épaisseur d'altération), 12,6\% (densité de drainage), 7,4\% (lithologie) et $6,5 \%$ (pente). La synthèse des six paramètres dépeint la répartition spatiale du potentiel en eau souterraine du bassin. Ainsi, 27,2 \% de la superficie totale du bassin affiche un potentiel très faible à faible. Les zones ayant un potentiel très faible $(9,2 \%)$ sont localisées vers Djidja, Gangan et les environs de Glazoué (Figure 7). Il s'agit des zones à faible hauteur de pluies, faible densité de fractures et ayant une épaisseur d'altérites faible à moyenne. L'aire occupée par le potentiel en eau souterraine bon à très bon atteint 48,2 \% . Les communes de Savalou, Dassa et Bantè affichent de bon potentiel en eau souterraine $(27,5 \%)$; cependant la fréquence des forages négatifs révèlent le caractère discontinu et aléatoire de la productivité hydrodynamique des aquifères. Toutefois les paramètres géologique (fractures, recouvrement), climatique (pluie) et topographique (drainage, pente) exploités dans cette étude, offrent un cadre favorable à l'identification des eaux souterraines. Les 
localités de Yagbo et Konkondji appartiennent à la zone modérée qui occupe $24,7 \%$ du secteur d'étude.

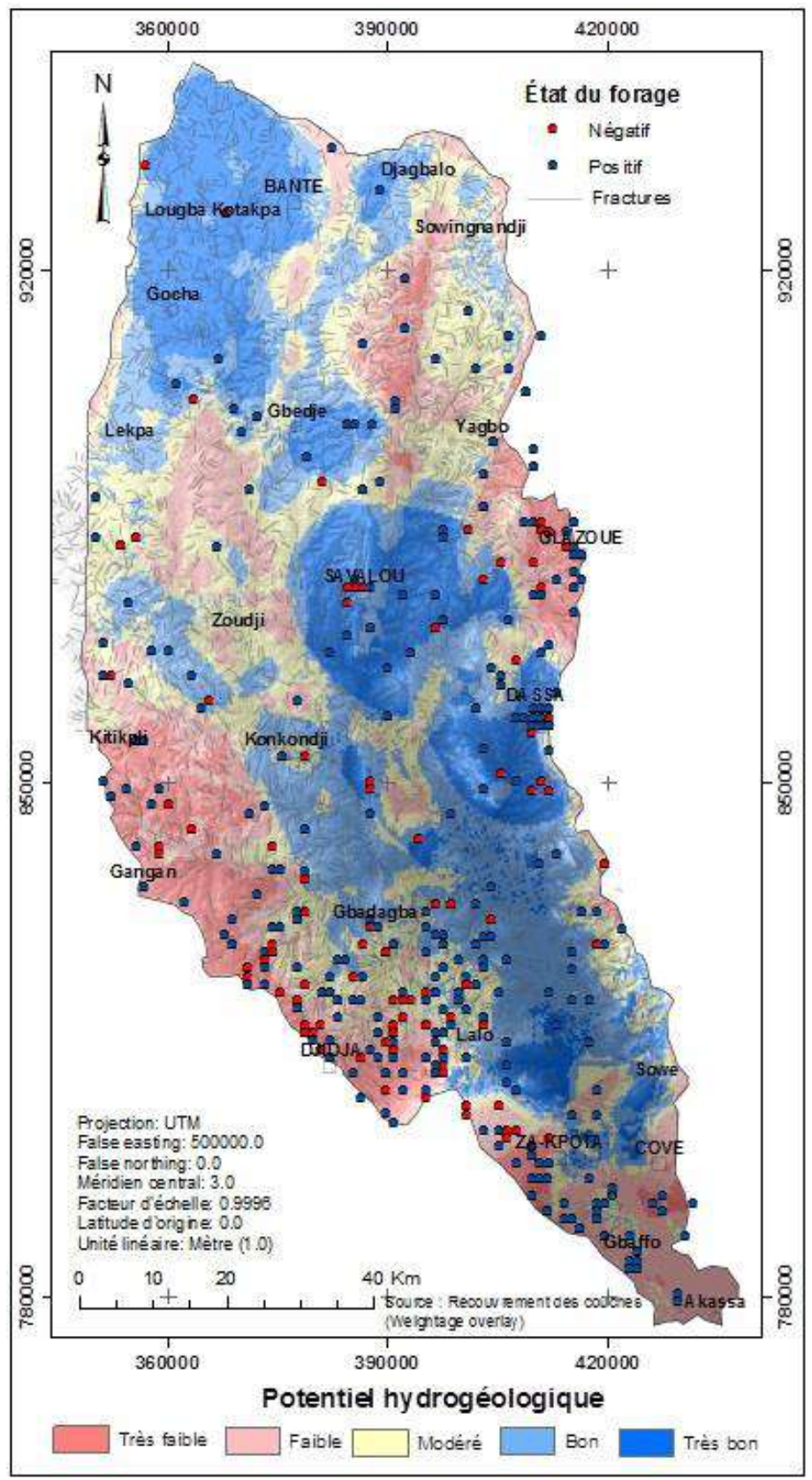

Figure 7. Carte du potentiel en eau souterraine

Les forages existant dans le bassin sont principalement des ouvrages d'hydrauliques villageoises tels que les forages équipés de pompe à motricité humaine (FPM), les postes d'eau autonomes (PEA) et les adductions d'eau 
villageoises (AEV). En moyenne, l'intervalle de $40 \mathrm{~m}$ à $60 \mathrm{~m}$ de profondeur suffit dans la plupart des zones pour obtenir un débit acceptable (plus de 500 forages). Cependant les réservoirs affichent deux conditions extrêmes. Soit le forage est profond (autour de $80 \mathrm{~m}$ ) et le débit n'augmente pas avec la profondeur (productivité limitée) : dans ce cas le forage prend généralement fin dans une fracture sèche ou faiblement productive. Soit le forage est moins profond (autour de $30 \mathrm{~m}$ ) et productif : le forage capte un réseau dense de fracture.

Le débit final d'un forage positif est subjectif car l'implantation dépend surtout de la demande en eau et de la position géographique de l'agglomération à desservir. La distribution spatiale indique que plus de $93 \%$ des forages sont situés à moins de $1 \mathrm{~km}$ d'une agglomération. Sauf dans le cas d'un piézomètre, l'opérateur généralement arrête la foration selon que le débit escompté est atteint. Un forage est par contre déclaré négatif quand tout espoir d'avoir le débit minimal requis est perdu révélant les limites hydrogéologiques de l'aquifère. Dans ce contexte, l'ouvrage représente un indicateur fiable pour l'appréciation du potentiel en eau souterraine (Figure 8).

La superposition de la Base de Donnée Intégrée (BDI) de la Direction Générale des Ressources en Eau (DGRE) avec la carte du potentiel en eau souterraine indique que 75 ouvrages sur 180 négatifs ciblés se localisent dans une zone de potentiel très faible à faible (Figure 7). Par ailleurs, dans les zones à potentiel en eau souterraine moyen, 46 forages négatifs sont identifiés et 59 dans les zones caractérisées par un potentiel bon à très bon. Cette tendance baissière d'échec vers les zones à forts potentiels valide le résultat de la figure 7.

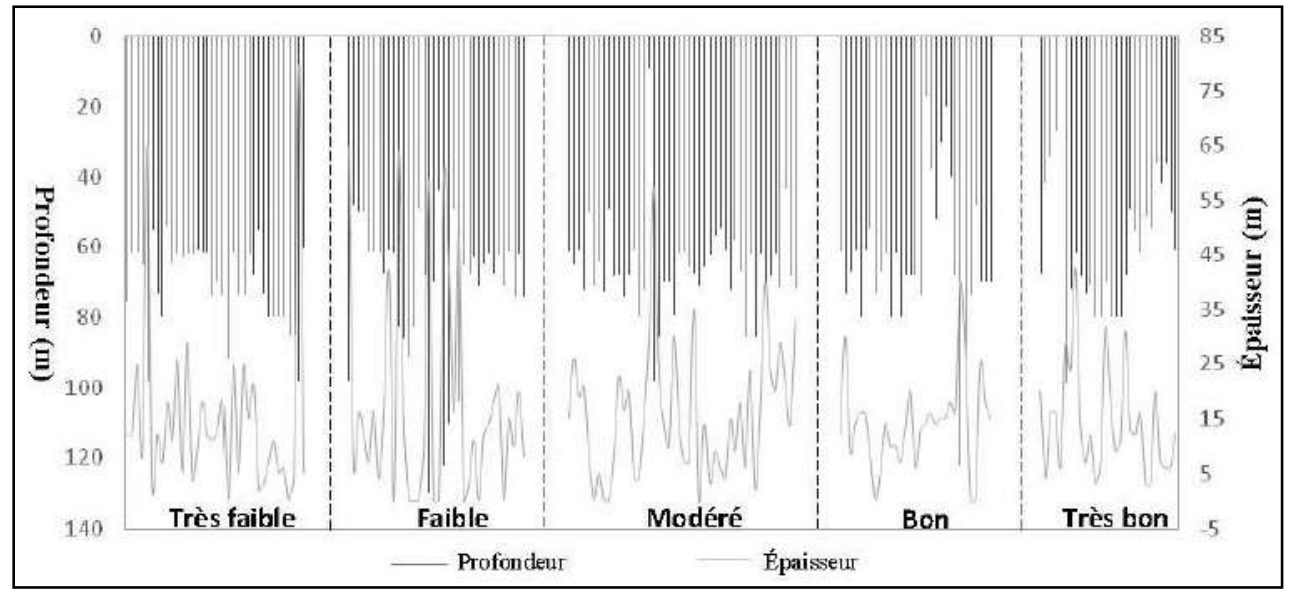

Figure 8. Profondeur de forage négatif et épaisseur d'altération versus potentiel en eau

\section{Discussion}

souterraine.

La disponibilité et la bonne qualité des eaux souterraines rendent la ressource relativement propice à l'AEP des populations. Particulièrement dans 
le bassin du Zou, les réservoirs d'eau souterraine sont séparés en aquifère de socle occupant la majeure partie du bassin et du sédimentaire situé au Sud. Dans les études traitant de l'analyse de la disponibilité des eaux souterraines, la nature de l'aquifère et les conditions hydro-climatiques rentrent en ligne de compte pour apprécier la pérennité des aquifères. Le réseau de fractures identifiées dans ce travail a révélé trois importantes directions : les orientations N180-N30 (25,8 \%), N90-N110 (17,3\%) et N60-N70 (11,1\%). La première famille se traduit par les fractures de la faille de Kandi (Guirauld et Alidou, 1981) connue sous le nom de la faille de Kidal-Takellout (Ball et Caby, 1984) au Mali et sous formes de fractures de direction N0 ayant affecté et sectionné les filons doléritiques du Sud-Ouest de la Côte d'Ivoire (Faillat, 1986 cité par Biémi, 1992). De plus, les travaux de Boukari (1982) ont reconnu à l'Est du secteur d'étude à l'aide des photographies aériennes des directions principales NNE-SSW et ESE-WNW. La seconde famille est connue depuis l'orogenèse panafricaine (N90-N100) et la dernière assimilable aux fractures du couloir tectonique de grande envergure qui l'aurait précédé (Biémi, 1992). La direction minoritaire N130-N140 (9,6 \%) est remarquable parmi les orientations identifiées. La reconnaissance des réseaux de fracture dense maximise la chance d'obtenir de bons débits. La carte de densité de fractures est un matériel de choix lors des campagnes hydrauliques en milieu de socle. La densité de fracture reconnue dans ce cas oscille entre 0,8 et 5,2 $\mathrm{km} / \mathrm{km}^{2}$ avec une moyenne de $2,4 \mathrm{~km} / \mathrm{km}^{2}$ et un écart type de $0,9 \mathrm{~km} / \mathrm{km}^{2}$. Les zones à fortes densité peuvent souvent être recouvertes d'épaisse couche d'altération et entretenir de très bons écoulements souterrains. De ce point de vue, on constate que près de plus de $60 \%$ de forages situés à moins de $200 \mathrm{~m}$ d'un linéament identifié est positif.

La densité de fractures contribue au calcul de la carte de potentiel à $27,5 \%$, l'épaisseur d'altération à $15,8 \%$ et la lithologie à 0,074 . La couche d'altération compte tenu de la nature de la roche saine sous-jacente renforce la disponibilité de l'eau si elle est importante. Le drainage (12,6\%), la topographie $(6,5 \%)$ et la pluviométrie (30,2\%) conditionnent aussi le potentiel en eau du bassin. A l'instar des travaux (Sreedhar et al., 2009 ; Abdalla, 2012 ; Kanohin et al., 2012 ; Magesh et al., 2012 ; Ndatuwong et Yadav, 2014 ; Oikonomidis et al., 2015) effectués un peu partout dans le monde, l'analyse multicritère de Saaty (1971) est validée avec un ratio de cohérence inférieur à $10 \%$ et appliquée aux six couches. Cet environnement de comparaison et de pondération a permis de dépeindre l'état des lieux de la disponibilité des ressources en eau souterraine dans un bassin. Ainsi, l'état des lieux du bassin indique un potentiel très faible de 9,2 \% vers Djidja, au Nord de Gangan et au Nord-Ouest de Yagbo, faible (18\%), modéré $(24,7 \%)$, bon $(27,5 \%)$ et très bon $(20,6 \%)$ dans les environs de Savalou, Dassa, Akassa et au Nord-Ouest de Bantè. La carte hydrogéologique du Bénin au 1/500 000 
réalisée par l'entreprise Allemande Ingenieur-Gesellschaft fur Internationale Planungsaufgaben $\mathrm{mbH}$ (IGIP) a révélé que la potentialité des eaux souterraines est médiocre à moyenne dans le secteur. Ce résultat obtenu depuis 2012 bien qu'étant de petite échelle corrobore également la présente étude dans la mesure où plus de $50 \%$ de la superficie du bassin affiche une potentialité très faible à moyenne.

\section{Conclusion}

En définitive, l'analyse du potentiel en eau souterraine fait appel à nombre de paramètres étudiés selon le contexte hydrogéologique. La technique d'analyse multicritère de décision (ratio de cohérence inférieur $10 \%$ ) rend à la carte finale une crédibilité indéniable. Dans le bassin du Zou, les aquifères affichent un potentiel très faible de 9,2 \%, faible (18 \%), modéré (24,7 \%), bon $(27,5 \%)$ et très bon $(20,6 \%)$. L'apport des fractures de direction majoritaire (N180-N30, N90-N110 et N60-N70) et de l'altération accroit considérablement le potentiel par endroit. Les directions minoritaires notamment la famille N130-N140 (9,6 \%) joue un rôle de découpage en bloc de ces aquifères de fractures. Ces résultats indiquent combien le bassin exige d'attention en cas d'implantation future de forages. Dans une perspective de mieux implanter les ouvrages à venir, une analyse détaillée de la ressource à des échelles plus grandes s'avère nécessaire afin de garantir un bon débit lors des campagnes d'hydraulique villageoise.

\section{References :}

1. Abdalla F. (2012). Mapping of groundwater prospective zones using remote sensing and GIS techniques: A case study from the Central Easten Desert, Egypt. Journal of African Earth Sciences : 70 (2012) 817.

2. Alidou S., Germain P., Julien J.L. et Tempier P. (1975). Sur le métamorphisme des quartzites du groupe de Badagba (Dahomey). C.R.Acad.Sc. 281, D, 339-342.

3. Alhassane I., Maman Sani A.B., Issoufou S., Bachir S.M., Boureima O. (2018). Apport des images ETM+ et du Modèle Numérique de Terrain (MNT) a la cartographie des fractures En région montagneuse : secteur de Timia (Massif de l'Aïr, Nord du Niger). European Scientific Journal March 2018 edition Vol.14, No.9 ISSN: 1857 - 7881 (Print) e - ISSN 1857- 7431. Doi: 10.19044/esj.2018.v14n9p103

4. Assoma V.T., Koudou A., Adiaffi B., Kouamé K.F. (2018). Caractérisation des structures géologiques par analyse morphostructurale d'image d'ombrage (ASAR ENVISAT et MNT SRTM) et géostatistique du sud de la Côte d'Ivoire. European 
Scientific Journal May 2018 edition Vol.14, No.15 ISSN: 1857 - 7881 (Print) e - ISSN 1857- 7431. Doi: 10.19044/esj.2018.v14n15p197

5. Awoyé R. (2007). Modélisation du bilan hydrologique du bassin versant du Klou : contribution à la gestion durable des ressources en eau dans le Zou. Thèse d'ingénieur FSA/UAC, Bénin, p. 100.

6. Ball E. et Caby R. (1984). Open folding and wrench movements. Their relationship with horizontal tectonics in the pan-african belt of northen Mali'. Geol. Afric. Fervuren.

7. Banton O. et Bangoy M. (1997). Hydrogéologie, multiscience environnementale des eaux souterraines. AUPELF-UREF et Presses de l'Université du Québec.

8. Becker M.W. (2006). Potential for satellite remote sensing of groundwater. Ground Water 2006 : 44(2) :306-18.

9. Biémi J. (1992). Contribution à l'étude géologique, hydrogéologique et par Télédétection des bassins versants subsahéliens du socle précambrien d'afrique de l'Ouest Hydrostructurale, hydrodynamique, hydrochimie et isotopie des aquifères discontinus des sillons et aires granitiques de la Haute Marahoué (Côte d'Ivoire). Thèse d'Etat Université Nationale de Côte d'Ivoire.

10. Boko M. (2004): Gestion des risques hydro-climatiques et développement économique durable dans le bassin du Zou. Rapport d'étude Cotonou Bénin. 51p.

11. Bossa Y.A. (2007) : Modélisation du bilan hydrologique dans le bassin du Zou à l'exutoire d'Atchérigbé: Contribution à l'utilisation durable des ressources en eau. Thèse d'ingénieur FSA/UAC, Bénin.

12. Boukari M. (1982). Contribution à l'étude hydrogéologique des régions de socle de l'Afrique intertropicale : l'Hydrogéologie de la région de Dassa-Zoume (Bénin). Thèse 3ème cycle, Université de Dakar, Sénégal, p173.

13. Boukari M. (1998). Fonctionnement du système aquifère exploité pour l'Approvisionnement en Eau de la ville de Cotonou sur le littoral Béninois. Impact du développement urbain sur la qualité des ressources. Thèse 3ème cycle, Université cheik Anta Diop, Dakar, Sénégal, p256.

14. Boussari W.T. (1975). Contribution à l'étude géologique du socle cristallin de la zone mobile pan-africaine (région centrale du Dahomey) . Thèse $3^{\mathrm{e}}$ cycle, Univ. Besançon.

15. Bruning J.N., Gierke J.S. \& Maclean A.L. (2009). Digital processing and data compilation approach for using remotely sensed imagery to identify geological lineaments in hard-rock terrains: an application for groundwater explorations in Nicaragua. ASPRS 2009 Annual Conference Baltimore, Maryland, March 9-13, 2009 
16. Burgeap-Brgm. (1994). Projet d'alimentation en eau potable des zones lacustres des départements de l'Atlantique et l'Ouémé. Rapport final, p3-25.

17. Colloque de Géologie Africaine. (2006). $21^{\text {ème }}$ Colloque de Géologie Africaine. Maputo, Mozambique, Juillet 2006.

18. Castany G. (1982). Principe et méthodes de l'hydrogéologie. Dunod, Paris, $238 \mathrm{p}$.

19. Chevarie P. \& Periche N. (2001). Application de la méthode AHP lors de l'utilisation d'un type de devis à obligation de performance. École de technologie supérieure, Montréal

20. Chorowicz J. (1984). Importance of pattern recognition for geological remote sensing applications and new look at geological maps. In: Remote sensing for geological Mapping, Téléki \& weber eds, documents BRGM, 82, p29-56.

21. Dewandel B., Lachassagne P., Wyns R., Marechal J.C., \& Krishnamurthy, N.S. (2006). A generalized hydrogeological conceptual model of granite aquifers controlled by single or multiphase weathering. Journal of Hydrology, 330, 260-284, doi:10.1016/j.jhydrol.2006.03.026

22. DG-Eau. (2012). Carte hydrogéologique du Bénin au 1/500000. Direction Générale de l'eau 2012.

23. Faillat J.P. (1986). Hétérogénéité et effet d'échelle dans les aquiferes fissurés. Approche par pompages d'éssai sur station expérimentale (Afrique de l'Ouest). BRGM, hydrogéol, n1, pp 65-76.

24. Guiraud R. et Alidou S. (1981). La faille de Kandi (Bénin), témoin du rejet fini-Crétacé d'un accident majeur a l'échelle de la plaque africaine .C.R. Acad. Sci., 293, 779-782, Paris.

25. Houssou S. C. (1998). Les bioclimats humains de l'Atacora (nordouest du Bénin) et leurs implications socio-économiques. Thèse de doctorat nouveau régime, Université de Bourgogne, Dijon, p322.

26. Jarvis A., Rubiano J., Nelson A., Farrow A. \& Mulligan M. (2004). Practical Use of SRTM Data in the Tropics: Comparisons with Digital Elevation Models Generated from Cartographic Data. Working Document vol. 198, International Centre for Tropical Agriculture (CIAT), Cali, Columbia.

27. Jourda R.J. P. (1987) Contribution à l'étude géologique et hydrogéologique de la région du Grand Abidjan (Côte d'Ivoire). Thèse Dr. 3è cycle, Univ. Grenoble 1, Inst. Dolomieu, 1987.

28. Kamagate B., Gone D. L., Doumouya I., Ouattara I., Ouedraogo M., Bamba A. \& Savane I. (2011). Relation nappe-rivière dans le bassin versant du Bandama en milieu de socle fissuré en Côte d'Ivoire : 
approche couplée hydrochimie - télédétection. Int. J. Biol. Chem. Sci. 5(1): 206-216, February 2011, ISSN 1991-8631.

29. Kanohin F. O., Saley M. B., Aké G. E. \& Savané I. (2012). Apport de la télédétection et des SIG dans l'identification des ressources en eau souterraine dans la région de Daoukro (Centre-Est de la Côte d'Ivoire). International Journal of Innovation and Applied Studies, 2012, 1 (1), pp.35-53. <hal-00771901>

30. Kedowide Mevo Guezo C. G. (2011). SIG et analyse multicritère pour l'aide à la décision en agriculture urbaine dans les pays en développement, cas de Ouagadougou au Burkina Faso. Thèse de Doctorat, Université Paris 8 Vincennes - Saint-Denis. 281p+Annexes

31. Kodja D., Vissin E. W. \& Amoussou E. (2012). Prévisibilité des hautes eaux avec le modèle conceptuel GR2M dans le bassin-versant du Zou au Benin. (Afrique l'ouest), In les climats régionaux : observation et modélisation. Actes du XXVème colloque de l'AIC, Grenoble, France 415-420

32. Kouamé K.F., Lasm T., Saley M.B., Tonyé E., Bernier M. \& Wasde S. (2009). Extraction linémentaires par morphologie mathématique sur une image RSO de RADARSAT-1 : application au socle Archéen de la Côte d'Ivoire. Journées d'Animation Scientifique (JAS09) de l'AUF, Alger Novembre 2009.

33. Kouassi E.A., Koffi Y. B., Kouassi A. M., Soro G., Soro N. \& Biemi J. (2013). Fonctionnement hydrodynamique des aquifères discontinus de la région d'Abidjan-Agboville (Sud de la Côte D'ivoire). International Journal of Geography and Geology, 2013, 2(5):52-69

34. Kouassi W.F., Kouassi K.A., Mangoua M.J., Kamenan Y.M., and Kouadio Z.A. (2019). Evaluation des potentialités en eau souterraine de la portion Ivoirienne du bassin versant du Niger. International Journal of Innovation and Applied Studies ISSN 2028-9324 Vol. 25 No. 2 Jan. 2019, pp. 774-784 (C) 2019 Innovative Space of Scientific Research Journals

35. Kouassi K.A., Kouassi F.W., Kouadio Z.A., Ballo Bi H.E., Coulibaly A. et Kamagaté B. (2018). Etude des anomalies de résistivités pour l'optimisation des implantations de forages d'hydraulique villageoise en milieu de socle: cas de la région du Haut-Sassandra (Centre-Ouest de la Côte d'Ivoire). European Scientific Journal August 2018 edition Vol.14, No.24 ISSN: 1857 - 7881 (Print) e-ISSN 1857- 7431. Doi: 10.19044/esj.2018.v14n24p69

36. Le Barbé L., Ale G., Millet B., Borel Y. \& GUALDE R. (1993). Les ressources en eaux superficielles de la République du Bénin. ORSTOM Editions, Paris. 
37. Magesh N. S., Chandrasekar N. \& Soundranayagam J. P. (2012). Delineation of groundwater potential zones in Theni district, Tamil Nadu, using remote sensing, GIS and MIF techniques. Geoscience Frontiers. doi:10.1016/j.gsf.2011.10.007

38. Meijerink A.M.J. (2007). Remote Sensing applications to groundwater. IHP-VI Series on Groundwater No. 16. UNESCO, Paris.

39. Ndatuwong L. G. \& Yadav G. S. (2014). Integration of Hydrogeological Factors for Identification of Groundwater Potential Zones Using Remote Sensing and GIS Techniques. Journal of Geosciences and Geomatics, 2014, Vol. 2, No. 1, 11-16. doi:10.12691/jgg-2-1-2

40. Oikonomidis D., Dimogianni S., Kazakis N. \& Voudouris K. (2015). A GIS/Remote Sensing-based methodology for groundwater potentiality assessment in Tirnavos area, Greece. Journal of Hydrology 525 (2015) 197-208. C) Elsevier B.V.

41. Okioh L. (1972). Contribution à l'étude morphologique des reliefs résiduels de la région de Dassa-Zoumé, Fita et Savalou (Dahomey). Thèse de doctorat, Université de Paris VII, Paris, 163 p.

42. Opricovic S. \& Tzeng G.-H. (2006). Extended VIKOR method in comparison with outranking methods. European Journal of Operation Research, 2006.

43. Rabus B., Eineder M., Roth A. \& Bamler R. (2003). The shuttle radar topography mission - a new class of digital elevation models acquired by spaceborne radar. ISPRS Journal of Photogrammetry and Remote Sensing, 57 (4), 241-262.

44. Sintondji L. O., Agbossou E.K. \& Degnissode B. (2013). Dynamique de dégradation des forêts galeries et comblement du cours d'eau Agbado dans le département des Collines au Bénin. Int. J. Biol. Chem. Sci. 7(4): 1555-1567, August 2013, issn 1991-8631.

45. Saaty L. (1971). How to make a decision: The Analytic Hierarchy Process, European of Operation Research, European journal of operational research ISSN 0377-2217 CODEN EJORDT. Vol. 48, pp. 9-26

46. Sreedhar Ganapuram G.T., Vijaya Kumar I.V., Murali K., Ercan K., Cüneyd Demirel M., (2009). Mapping of groundwater potential zones in the Musi basin using remote sensing data and GIS. Advances in Engineering Software: 40 (2009) 506-518

47. Vision Eau Bénin. (1999). Vision Eau Bénin 2025. Document final, Décembre 1999.

48. Vissin E. W. (1998). Contribution à l'étude du fonctionnement hydrologique du bassin de la Sota . Mémoire de Maîtrise, FLASH/UAC, 80p. 
49. Youan TA M., LASM T., Jourda J.P., Kouamé K.F. \& Moumtaz R. (2008). Cartographie des accidents géologiques par imageries satellitaire Landsat-7 ETM+ et analyse des réseaux de fractures du socle précambrien de la région de Bondoukou (Nord-Est de la Côte d'Ivoire). Télédétection, Editions scientifiques GB, 2008, 8 (2), pp119135. <halshs-00392312> 